\title{
Nonlinear Complex Dynamics of Carbon Emission Reduction Cournot Game with Bounded Rationality
}

\author{
LiuWei Zhao \\ Computational Experiment Center for Social Science, School of Management, Jiangsu University, Zhenjiang, Jiangsu 212013, China \\ Correspondence should be addressed to LiuWei Zhao; 136901672@qq.com
}

Received 8 July 2017; Revised 14 August 2017; Accepted 10 September 2017; Published 12 October 2017

Academic Editor: Viet-Thanh Pham

Copyright (C) 2017 LiuWei Zhao. This is an open access article distributed under the Creative Commons Attribution License, which permits unrestricted use, distribution, and reproduction in any medium, provided the original work is properly cited.

\begin{abstract}
Based on the hypothesis of participant's bounded rationality, our study formulated a novel Cournot duopoly game model of carbon emission reduction and, subsequently, analyzed the dynamic adjustment mechanism of emission reduction for enterprises. The existence and stability of the equilibrium solution of game are further discussed by the nonlinear dynamics theory. Our findings revealed that the parameters have key significance on the dynamic properties of the system. However, when the adjustment speed gets too large, the system loses the original stability and vividly demonstrates complex chaos phenomenon. Higher market prices in carbon trading have an outstanding impact on the stability of the system, which easily leads to system instability. Our study further controlled the chaos behavior of the power system by the delay feedback control. The results of the numerical analysis depict that the unstable behavior of the dynamic system can be controlled efficiently and quickly, in the quest to restore back a stable and orderly market. Our novel method is proved to have provided decision makers with effective solution to market instability.
\end{abstract}

\section{Introduction}

Massive economic growth has resulted in severe environmental issues such as environmental pollution and global climate warming. There is, therefore, huge concerns arising from the United Nations Framework Convention on Climate Change (UNFCCC) and various countries, to develop a series of related policies and regulations to curb these environmental menaces. Carbon emission trading (carbon trading for short) is one of the key market mechanism approaches used to reduce global greenhouse, thus carbon dioxide emission reduction. On 9 May 1992, an environmental treaty on the issue of climate change was negotiated and passed by international governments and the United Nations Framework Convention. In December 1997 in Tokyo, Japan, the "Kyoto Protocol" was issued. The Kyoto Protocol launched a market mechanism as a new solution to the problems of greenhouse gas emission reduction with a focus on the reduction of carbon dioxide since carbon emission has been the principal greenhouse gas. This entails trading carbon as a commodity: emitting carbon dioxide beyond the limits generates a potential cost; and conversely, those who are within a defined scope hold a potential value.
Despite the fact that China is a developing country and not subject to stricter obligation of reducing emissions under the Kyoto Protocol, this global climate changes and the carbon trading in developed countries around the world has brought tremendous changes in China. Consequently, China is playing an active role in the establishment of carbon trading pilots in larger cities, such as Beijing, Shanghai, and other provinces and cities. China has already initiated such pilot projects and far advanced in its implementation. The main participants in the carbon trading market are the industrial enterprises. This carbon trading impacts on enterprises' production processes, technology, profits, and other critical aspects. Therefore, it is of great significance to study and analyze the influence of carbon trading on the production, decision-making, and profits of the enterprise, which would provide sufficient theoretical basis for the perfection and implementation of the right carbon trading policy.

The main concerns about carbon emissions trading can be categorized into three aspects: firstly, the initial allocation of carbon emissions rights [1,2]; secondly, the price of carbon emissions trading [3]; and thirdly the economic benefits arising from carbon emissions trading [4]. With regard to 
the development of carbon emissions trading, the industry structure of the enterprises has a great impact on the carbon trading. For instance, in the oligopolistic industry market, the carbon trading price is more susceptible to the impact of enterprises' transactions, in consequence, making the carbon emissions trading prices deviate from the market's equilibrium price. Therefore, the research on the game behavior of carbon emissions trading in oligopoly market has become a vital problem that needs to be solved urgently.

The two classical models in the theory of oligopoly are those of Cournot $[5,6]$ and Bertrand $[7,8]$. In Cournot's model, the firms choose to compete on output quantity, and in Bertrand's model, they choose to compete on price. Both models can be interpreted as static games where decisions are made simultaneously and where each firm maximizes its own profit, in a context of perfect and complete information $[9,10]$. This paper introduces carbon emission trading in the classic Cournot output game framework and formulates a novel Cournot duopoly game model of carbon emission reduction based on the hypothesis of participant's bounded rationality. The dynamic adjustment mechanism of emission reduction is also analyzed in this paper. According to the hypothesis that the market inverse demand function and the cost function are linear functions and the participants have bounded rationality, our study constructs the nonlinear dynamic Cournot game model to analyze the local stability of the Nash equilibrium. The stability of the boundary equilibrium for the four-dimensional dynamical system is further analyzed based on the stability of the discrete system. Consequently, the parameters conditions of interior equilibrium and stability are obtained according to Schur-Cohn criterion. Based on the analysis of all equilibrium's stability of the nonlinear investment game system, our study adopts the numerical simulation results to analyze the influence of the model parameters on the stability and complexity of the dynamic system. The chaos behavior of the system is effectively controlled by the delayed feedback control method.

This article is organized as follows: in Section 2, the model is established based on the hypothesis of bounded rationality. In Section 3, we discuss the existence and local stability of the equilibrium points in the system. In Section 4 , we demonstrate the dynamic features of this system with numerical simulations, including bifurcation diagram, phase portrait, and sensitive dependence on initial conditions. In Section 5, delayed feedback control is utilized to stabilize the chaotic behaviors of the system.

\section{Model Hypotheses and Establishment}

Our study assumes that the two production enterprises develop and produce homogeneous products. Each enterprise's action strategy is to choose the amount of investment in emission reduction during various periods and meet the requirements: two participating companies develop the corresponding emission reduction investment strategy in the discrete timeline. The $K_{i}(t-1)$ reflects the company's capital stock in the period of the $t-1, x_{i}(t)$ for its single-stage carbon emission reduction in the period of $t$, and emission reduction cost coefficient is $\beta_{i}(\beta>0)$, so the enterprise's single-stage emission reduction investment is $\beta_{i} x_{i}(t)$. Due to the existence of a certain amount of capital depreciation, investment capital stock $K_{i}(t-1)$ flows into the next economic period, and it remains $\theta K_{i}(t-1)$, among which $\theta(0<\theta<1)$ stands for the residual ratio. Thus, we can reach that the relational expression in the adjacent two periods of investment capital stock of enterprise $i$ is

$$
K_{i}(t)=\theta K_{i}(t-1)+\beta_{i} x_{i}(t), \quad i=1,2 .
$$

For each particular enterprise's products amount in a certain period of time, production potential is determined by the accumulation amount of corporate investment capital in the period $K_{i}(t)$. Our study utilizes a linear form $q_{i}(t)=$ $B_{i} K_{i}(t)$ to represent [11], among which positive constant $B_{i}$ denotes the technological level of enterprises for innovative production on emerging products. Thus, we can reach that $q_{i}(t)=B_{i}\left(\theta K_{i}(t-1)+\beta_{i} x_{i}(t)\right), i=1,2$.

Our study assumes that the sales price of this kind of products on the market is a linear inverse demand function $p(t)=a-b Q(t), a, b>0$, and $Q(t)=q_{1}(t)+q_{2}(t)$ represents the sum of the two firms' supply in the market. At the same time, the firm's linear production cost function $C_{i}\left(q_{i}(t)\right)=$ $c_{i} q_{i}(t)(i=1,2)$, marginal $\operatorname{costs} c_{1}$ and $c_{2}$ are positive numbers.

The relationship between the amount of carbon dioxide produced by the enterprise during the production process and its production $q_{i}(t)$ is expressed as a linear relationship in the form of $k_{i} q_{i}(t) . k_{i}\left(k_{i}>0\right)$ is the coefficient of carbon dioxide produced by the enterprise $i$. Therefore, the enterprise's total carbon emission is $k_{i} q_{i}(t)-x_{i}(t)$ in the single period of $t$. Each enterprise in the $t$ period has a certain quota of carbon emissions rights- $y_{i}(t)$, and the enterprise's carbon emissions trading volume is $y_{i}(t)-\left(k_{i} q_{i}(t)-x_{i}(t)\right)$, and the market price of carbon trading is $P$. If $y_{i}(t)-\left(k_{i} q_{i}(t)-x_{i}(t)\right)>$ 0 , it means that the company sells the remaining carbon emissions; on the other hand, if the company needs to buy the lacking carbon emissions, $y_{i}(t)-\left(k_{i} q_{i}(t)-x_{i}(t)\right)=-\left(y_{j}(t)-\right.$ $\left.\left(k_{j} q_{j}(t)-x_{j}(t)\right)\right)$.

Based on the above assumptions about the function relationship among the variables, the profit of the enterprise $i$ in the period $t$ is calculated as follows:

$$
\begin{aligned}
\pi_{i}\left(x_{1}(t), x_{2}(t)\right)= & q_{i}(t)\left(p(t)-c_{i}\right)-\beta_{i} x_{i}(t) \\
& +P\left(y_{i}(t)-\left(k_{i} q_{i}(t)-x_{i}(t)\right)\right), \\
& i=1,2 .
\end{aligned}
$$

The specific expression of each variable is substituted into (2) and reaches first partial derivative about $x_{i}(t)$ on $\pi_{i}\left(x_{1}(t), x_{2}(t)\right)$, and the marginal profits of the two enterprises are

$$
\begin{aligned}
\varphi_{1}(t) & =\frac{\partial \pi_{1}\left(x_{1}(t), x_{2}(t)\right)}{\partial x_{1}(t)}=P \\
& +\beta_{1}\left(\begin{array}{c}
-b B_{1} B_{2}\left(\theta K_{2}(t-1)+\beta_{2} x_{2}(t)\right)+a B_{1}-c_{1} B_{1} \\
-P k_{1} B_{1}-1-2 b B_{1}^{2}\left(\theta K_{1}(t-1)+\beta_{1} x_{1}(t)\right)
\end{array}\right),
\end{aligned}
$$




$$
\begin{aligned}
\varphi_{2}(t) & =\frac{\partial \pi_{2}\left(x_{1}(t), x_{2}(t)\right)}{\partial x_{2}(t)}=P \\
& +\beta_{2}\left(\begin{array}{c}
-b B_{1} B_{2}\left(\theta K_{1}(t-1)+\beta_{1} x_{1}(t)\right)+a B_{2}-c_{2} B_{2} \\
-P k_{2} B_{2}-1-2 b B_{2}^{2}\left(\theta K_{2}(t-1)+\beta_{2} x_{2}(t)\right)
\end{array}\right) .
\end{aligned}
$$

In reality, due to the asymmetry of market information and the constraints of their own conditions, enterprises can generally reach only limited degree of rationality in the decision-making and cannot make a complete control on the future market demand; therefore, the two production enterprises will rely on their own local knowledge about the marginal profits to adjust the emission reduction strategies during the next period. In other words, if the business observes the positive marginal profit in the current period, then it will increase emission reduction in the period of $t+1$ and vice versa. As a result, the enterprise's dynamic adjustment mechanism can be expressed as

$$
x_{i}(t+1)=x_{i}(t)+\alpha_{i}\left(x_{i}(t)\right) \varphi_{i}(t), \quad i=1,2,
$$

where $\alpha_{i}\left(x_{i}(t)\right)$ is a positive adjustment function and reflects the company's adjustment range of emission reduction based on the marginal profits. Our study considers the form of the adjustment function in linear form as $\alpha_{i}\left(x_{i}(t)\right)=\alpha_{i} x_{i}(t)$, the coefficient $\alpha_{i}>0$ when the enterprise adjusts the rate of emission reduction strategy according to the marginal profit signal. Thus, (4) is

$$
x_{i}(t+1)=x_{i}(t)+\alpha_{i} x_{i}(t) \varphi_{i}(t), \quad i=1,2 .
$$

By connecting (1), (3a), (3b), and (5), in order to obtain the same expression by replacing $K_{i}(t-1)$ with $I_{i}(t)$, as shown from (6), we obtained a four-dimensional discrete dynamical model by replacing $K_{i}(t-1)$ with $I_{i}(t)$ as follows:

$$
\begin{aligned}
& x_{1}(t+1)=x_{1}(t)+\alpha_{1} x_{1}(t)[P \\
& \left.+\beta_{1}\left(\begin{array}{c}
-b B_{1} B_{2}\left(\theta I_{2}(t)+\beta_{2} x_{2}(t)\right)+a B_{1}-c_{1} B_{1} \\
-P k_{1} B_{1}-1-2 b B_{1}^{2}\left(\theta I_{1}(t)+\beta_{1} x_{1}(t)\right)
\end{array}\right)\right] \\
& x_{2}(t+1)=x_{2}(t)+\alpha_{2} x_{2}(t)[P \\
& \left.+\beta_{2}\left(\begin{array}{l}
-b B_{1} B_{2}\left(\theta I_{1}(t)+\beta_{1} x_{1}(t)\right)+a B_{2}-c_{2} B_{2} \\
-P k_{2} B_{2}-1-2 b B_{2}^{2}\left(\theta I_{2}(t)+\beta_{2} x_{2}(t)\right)
\end{array}\right)\right] \\
& I_{1}(t+1)=\theta I_{1}(t)+\beta_{1} x_{1}(t) \\
& I_{2}(t+1)=\theta I_{2}(t)+\beta_{2} x_{2}(t) .
\end{aligned}
$$

The discrete dynamical system (6) is based on the assumption that the market inverse demand function and the production cost function are all linear forms, describing the competition model of the emission reduction strategy between the two production enterprises. The game participants with bounded rational expectation will adjust the emission reduction strategy in different periods according to the marginal profits.

\section{Analysis of Equilibrium Point's Stability}

In the dynamical system $(6)$, let $x_{i}(t+1)=x_{i}(t)$ and $I_{i}(t+1)=$ $I_{i}(t)(i=1,2)$; we then get

$$
\begin{aligned}
& x_{1}(t)[P \\
& \left.\quad+\beta_{1}\left(\begin{array}{c}
-b B_{1} B_{2}\left(\theta I_{2}(t)+\beta_{2} x_{2}(t)\right)+a B_{1}-c_{1} B_{1} \\
-P k_{1} B_{1}-1-2 b B_{1}^{2}\left(\theta I_{1}(t)+\beta_{1} x_{1}(t)\right)
\end{array}\right)\right] \\
& \quad=0 \\
& x_{2}(t)[P \\
& \left.\quad+\beta_{2}\left(\begin{array}{l}
-b B_{1} B_{2}\left(\theta I_{1}(t)+\beta_{1} x_{1}(t)\right)+a B_{2}-c_{2} B_{2} \\
-P k_{2} B_{2}-1-2 b B_{2}^{2}\left(\theta I_{2}(t)+\beta_{2} x_{2}(t)\right)
\end{array}\right)\right] \\
& \quad=0 \\
& \frac{(1-\theta)}{\beta_{1}} I_{1}(t)=x_{1}(t) \\
& \frac{(1-\theta)}{\beta_{2}} I_{2}(t)=x_{2}(t) .
\end{aligned}
$$

In solving (7), we obtained the four equilibrium points of the dynamical system (6) as follows:

$$
\begin{aligned}
E_{0} & =(0,0,0,0), \\
E_{1} & =\left(\frac{(1-\theta)\left(P-\beta_{1}+B_{1} \beta_{1}\left(a-c_{1}-P k_{1}\right)\right)}{2 b B_{1}^{2} \beta_{1}^{2}}, 0,\right. \\
& \left.\frac{P-\beta_{1}+B_{1} \beta_{1}\left(a-c_{1}-P k_{1}\right)}{2 b B_{1}^{2} \beta_{1}}, 0\right), \\
E_{2} & =\left(0, \frac{(1-\theta)\left(P-\beta_{2}+B_{2} \beta_{2}\left(a-c_{2}-P k_{2}\right)\right)}{2 b B_{2}^{2} \beta_{2}^{2}}, 0,\right. \\
& \left.\frac{P-\beta_{2}+B_{2} \beta_{2}\left(a-c_{2}-P k_{2}\right)}{2 b B_{2}^{2} \beta_{1}}\right) \\
E^{*} & =\left(\frac{(1-\theta) W_{1}}{3 b B_{1}^{2} \beta_{1}^{2} B_{2} \beta_{2}}, \frac{(1-\theta) W_{2}}{3 b B_{1} B_{2}^{2} \beta_{1} \beta_{2}^{2}}, \frac{W_{1}}{3 b B_{1}^{2} B_{2} \beta_{1} \beta_{2}},\right. \\
& \left.\frac{W_{2}}{3 b B_{1} B_{2}^{2} \beta_{1} \beta_{2}}\right),
\end{aligned}
$$

where $W_{1}=2 B_{2}\left(P-\beta_{1}\right) \beta_{2}+B_{1} \beta_{1}\left(\beta_{2}\left(1+B_{2}\left(a-2 c_{1}+c_{2}-\right.\right.\right.$ $\left.\left.\left.2 P k_{1}+P k_{2}\right)\right)-P\right), W_{2}=B_{1} \beta_{1}\left(2 P+\left(B_{2}\left(a+c_{1}-2 c_{2}+P k_{1}-\right.\right.\right.$ $\left.\left.\left.2 P k_{2}\right)-2\right) \beta_{2}\right)-B_{2}\left(P-\beta_{1}\right) \beta_{2}$.

Easy to know, $E_{0}, E_{1}$, and $E_{2}$ are the boundary equilibrium points; $E^{*}$ is the only interior point equilibrium. Since the real economic significance of the system equilibrium point is taken into account, only the case when the equilibrium point is nonnegative is discussed. According to the assumption that the parameters $b, \beta_{1}, \beta_{2}, B_{1}, B_{2}$, and $\theta$ are positive parameters, so parameters need to meet the following conditions when $E_{1}, E_{2}$, and $E^{*}$ are greater than zero: 


$$
\begin{array}{r}
P-\beta_{1}+B_{1} \beta_{1}\left(a-c_{1}-P k_{1}\right)>0, \\
P-\beta_{2}+B_{2} \beta_{2}\left(a-c_{2}-P k_{2}\right)>0, \\
W_{1}>0, \\
W_{2}>0 .
\end{array}
$$

In the following analysis, the nonnegative condition (9) is assumed to be true.

3.1. Boundary Equilibrium Point Stability. To discuss the stability of the equilibrium points $\left(x_{1}, x_{2}, I_{1}, I_{2}\right)$ of the dynamical system (6), we first calculated the corresponding Jacobian matrix:

$$
J\left(x_{1}, x_{2}, I_{1}, I_{2}\right)=\left(\begin{array}{cccc}
1-\alpha_{1} M_{1} & -b B_{1} B_{2} x_{1} \alpha_{1} \beta_{1} \beta_{2} & -2 b \theta B_{1}^{2} x_{1} \alpha_{1} \beta_{1} & -b \theta B_{1} B_{2} x_{1} \alpha_{1} \beta_{1} \\
-b B_{1} B_{2} x_{2} \alpha_{2} \beta_{1} \beta_{2} & 1-\alpha_{2} M_{2} & -b \theta B_{1} B_{2} x_{2} \alpha_{2} \beta_{2} & -2 b \theta B_{2}^{2} x_{2} \alpha_{2} \beta_{2} \\
\beta_{1} & 0 & \theta & 0 \\
0 & \beta_{2} & 0 & \theta
\end{array}\right)
$$

where $M_{1}=\beta_{1}+2 b B_{1}^{2} \beta_{1}\left(\theta I_{1}+2 x_{1} \beta_{1}\right)-P-B_{1} \beta_{1}\left(a-c_{1}-P k_{1}-\right.$ $\left.b B_{2}\left(\theta I_{2}+x_{2} \beta_{2}\right)\right), M_{2}=\beta_{2}+2 b B_{2}^{2} \beta_{2}\left(\theta I_{2}+2 x_{2} \beta_{2}\right)-B_{2} \beta_{2}(a-$ $\left.c_{2}-P k_{2}-b B_{1}\left(\theta I_{1}+x_{1} \beta_{1}\right)\right)-P$.

According to the Schur-Cohn stability criterion [12], when all the eigenvalues of the characteristic polynomial corresponding to the Jacobian matrix (10) are in the unit circle on the complex plane, that is, the modulus of any eigenvalue is less than 1 , the equilibrium point $\left(x_{1}, x_{2}, I_{1}, I_{2}\right)$ is asymptotically stable.

Proposition 1. Boundary equilibrium point is unstable.

Proof. Substituting $E_{0}=(0,0,0,0)$ into the general formula (10) of the system Jacobian matrix, the Jacobi matrix of system (6) at the equilibrium point is expressed as follows:

$$
J\left(E_{0}\right)=\left(\begin{array}{c}
1+\alpha_{1}\left(\begin{array}{c}
B_{1}\left(a-c_{1}\right) \beta_{1}-\beta_{1} \\
+P\left(1-B_{1} k_{1} \beta_{1}\right)
\end{array}\right) \\
0 \\
\beta_{1} \\
0
\end{array}\right.
$$

We can reach four characteristic roots by calculation, namely,

$$
\begin{aligned}
& \lambda_{1}=\lambda_{2}=\theta, \\
& \lambda_{3}=1+\alpha_{1}\left(B_{1}\left(a-c_{1}\right) \beta_{1}-\beta_{1}+P\left(1-B_{1} k_{1} \beta_{1}\right)\right), \\
& \lambda_{4}=1+\alpha_{2}\left(B_{2}\left(a-c_{2}\right) \beta_{2}-\beta_{2}+P\left(1-B_{2} k_{2} \beta_{2}\right)\right) .
\end{aligned}
$$

From the nonnegative condition (9) of the equilibrium point and the adjustment coefficient $\alpha_{i}>0$, the latter two characteristic roots both satisfy $\left|\lambda_{3,4}\right|>1$. Therefore, the equilibrium point is unstable.

Proposition 2. $E_{1}$ and $E_{2}$ are unstable equilibrium points.

Proof. Jacobian matrix (10) in the specific form at the boundary equilibrium point is

$$
J\left(E_{1}\right)=\left(\begin{array}{cccc}
1+\alpha_{1}(1-\theta) H_{1} & \frac{(1-\theta) B_{2} \alpha_{1} H_{1} \beta_{2}}{2 B_{1} \beta_{1}} & \frac{(1-\theta) \theta \alpha_{1} H_{1}}{\beta_{1}} & \frac{(1-\theta) \theta B_{2} \alpha_{1} H_{1}}{2 B_{1} \beta_{1}} \\
0 & 1+\frac{\alpha_{2} W_{2}}{2 B_{1} \beta_{1}} & 0 & 0 \\
\beta_{1} & 0 & \theta & 0 \\
0 & \beta_{2} & 0 & \theta
\end{array}\right) \text {, }
$$

where $H_{1}=\left(1-B_{1}\left(a-c_{1}-P k_{1}\right)\right) \beta_{1}-P$. 
By calculating the eigenvalue of the Jacobian matrix $J\left(E_{1}\right)$, $\lambda_{1}=\theta, \lambda_{2}=1+\alpha_{2} W_{2} / 2 B_{1} \beta_{1}, \lambda_{3,4}=(1 / 2)\left(H_{2} \pm \sqrt{H_{2}^{2}-4 \theta}\right)$, where $H_{2}=1+\theta+(1-\theta) \alpha_{1}\left(\left(1-B_{1}\left(a-c_{1}-P k_{1}\right)\right) \beta_{1}-P\right)$.

From the previous parameters that $B_{1}, \alpha_{2}>0, \lambda_{2}>1$ can be pushed by the inequality equation (9). Thus, according to discrete dynamical system's equilibrium point stability determination conditions in the second chapter, we can know that $E_{1}$ is unstable boundary equilibrium. Similarly, the border equilibrium $E_{2}$ is also unstable.

3.2. Interior Point Equilibrium Stability. This section focuses on the stability of interior point equilibrium $E^{*}$. The Jacobian matrix (10) of interior point equilibrium $E^{*}$ is expressed as follows:

$$
J\left(E^{*}\right)=\left(\begin{array}{cccc}
1-\frac{2(1-\theta) \alpha_{1} W_{1}}{3 B_{2} \beta_{2}} & -\frac{(1-\theta) \alpha_{1} W_{1}}{3 B_{1} \beta_{1}} & \frac{2(1-\theta) \theta \alpha_{1} W_{1}}{3 B_{2} \beta_{1} \beta_{2}} & -\frac{(1-\theta) \theta \alpha_{1} W_{1}}{3 B_{1} \beta_{1} \beta_{2}} \\
-\frac{(1-\theta) \alpha_{2} W_{2}}{3 B_{2} \beta_{2}} & 1-\frac{2(1-\theta) \alpha_{2} W_{2}}{3 B_{1} \beta_{1}} & -\frac{(1-\theta) \theta \alpha_{2} W_{2}}{3 B_{2} \beta_{1} \beta_{2}} & \frac{2(1-\theta) \theta \alpha_{2} W_{2}}{3 B_{1} \beta_{1} \beta_{2}} \\
\beta_{1} & 0 & \theta & 0 \\
0 & \beta_{2} & 0 & \theta
\end{array}\right) .
$$

The characteristic polynomial of the matrix $J\left(E^{*}\right)$ is given as $P(\lambda)$, and $P(\lambda)=\lambda^{4}+p_{1} \lambda^{3}+p_{2} \lambda^{2}+p_{3} \lambda+p_{4}$, and the polynomial coefficients by the numerical calculation are as follows:

$$
\begin{aligned}
& p_{1}=-2\left(1+\theta-\frac{(1-\theta)}{3}\left(\frac{W_{2} \alpha_{2}}{3 B_{1} \beta_{1}}+\frac{W_{1} \alpha_{1}}{B_{2} \beta_{2}}\right)\right), \\
& p_{2}=\frac{\left(\begin{array}{c}
(-1+\theta) W_{1} \alpha_{1}\left(2(1+3 \theta) B_{1} \beta_{1}-(1-\theta) W_{2} \alpha_{2}\right) \\
+B_{2}\left(3\left(1+4 \theta+\theta^{2}\right) B_{1} \beta_{1}-2\left(1+2 \theta-3 \theta^{2}\right) W_{2} \alpha_{2}\right) \beta_{2}
\end{array}\right)}{3 B_{1} B_{2} \beta_{1} \beta_{2}},
\end{aligned}
$$

$$
\begin{aligned}
P(1) & =1+p_{1}+p_{2}+p_{3}+p_{4}=\frac{\alpha_{1} \alpha_{2}(\theta-1)^{2} W_{1} W_{2}}{3 B_{1} B_{2} \beta_{1} \beta_{2}}>0, \\
P(-1) & =1-p_{1}+p_{2}-p_{3}+p_{4}=\frac{\left[B_{1} B_{2} \beta_{1} \beta_{2}\left(\begin{array}{c}
4(1+\theta)\left(3(1+\theta)-N_{1}\right) \\
-(1-\theta) \alpha_{1}\left(P-\beta_{1}\right)\left(8(1+\theta)-N_{2}\right)
\end{array}\right)+\left(\begin{array}{c}
2(1-\theta) B_{2}^{2} \alpha_{2}\left(2(1+\theta)-(1-\theta) \alpha_{1}\left(P-\beta_{1}\right)\right) \\
\left(P-\beta_{1}\right) \beta_{2}^{2}+B_{1}^{2} \beta_{1}^{2} N_{3}\left(4(1+\theta)-N_{1}\right)
\end{array}\right)\right]}{3 B_{1} B_{2} \beta_{1} \beta_{2}}>0, \\
1 \pm p_{4} & =1 \pm \theta^{2}>0,
\end{aligned}
$$

From the Schur-Cohn stability criterion [12], if there is an associated characteristic polynomial $P(\lambda)$, that is, all eigenvalues of the Jacobian matrix $J\left(E^{*}\right)$ are located in the unit circle on the complex plane, then the coefficients of the polynomial need to satisfy the conditions in the Schur-Cohn stability criteria at the same time. The system parameters are added to it and simplified: where $N_{1}=(1-\theta) \alpha_{2}\left(2 P+\left(B_{2}\left(a+c_{1}-2 c_{2}+P k_{1}-2 P k_{2}\right)-\right.\right.$ 2) $\left.\beta_{2}\right), N_{2}=(1-\theta) \alpha_{2}\left(5 P+\left(B_{2}\left(a+4 c_{1}-5 c_{2}+4 P k_{1}-5 P k_{2}\right)-\right.\right.$ 5) $\left.\beta_{2}\right), N_{3}=(1-\theta) \alpha_{1}\left(P-\left(1+B_{2}\left(a-2 c_{1}+c_{2}-2 P k_{1}+P k_{2}\right)\right) \beta_{2}\right)$.

From the positive and negative assumptions of the system parameters and inequality (9), we can learn that the stability conditions $P(1)>0$ and $1 \pm p_{4}>0$ are tenable for sure. Therefore, the sufficient conditions for the asymptotic stability of the internal point equalization $E^{*}$ of the dynamical system (6) are classified as follows.

When $P(-1)=1-p_{1}+p_{2}-p_{3}+p_{4}>0$ and $\left|M_{3}^{ \pm}\right|>0$,

$$
\begin{aligned}
& p_{1}+p_{3}<1+p_{2}+p_{4} \\
& p_{2}-p_{2} p_{4}+p_{4}-p_{4}^{3}-p_{1} p_{3}+p_{1}^{2} p_{4}
\end{aligned}
$$

$$
<\left|1-p_{4}^{2}+p_{2} p_{4}-p_{2} p_{4}^{2}+p_{1} p_{3} p_{4}-p_{3}^{2}\right|
$$

All the eigenvalues of $J\left(E^{*}\right)$ satisfy the $\left|\lambda_{i}\right|<1(i=1,2,3,4)$, so that the equilibrium point $E^{*}$ is stable when the condition (17) is satisfied. From the above equilibrium point analysis, we can see that the stability of the known equilibrium points is dependent on the value of the system parameters.

\section{Numerical Simulation}

In order to intuitively analyze the dynamic behavior of model (6), we used the numerical simulation to visually describe the dynamic evolution process of the discrete dynamical system 

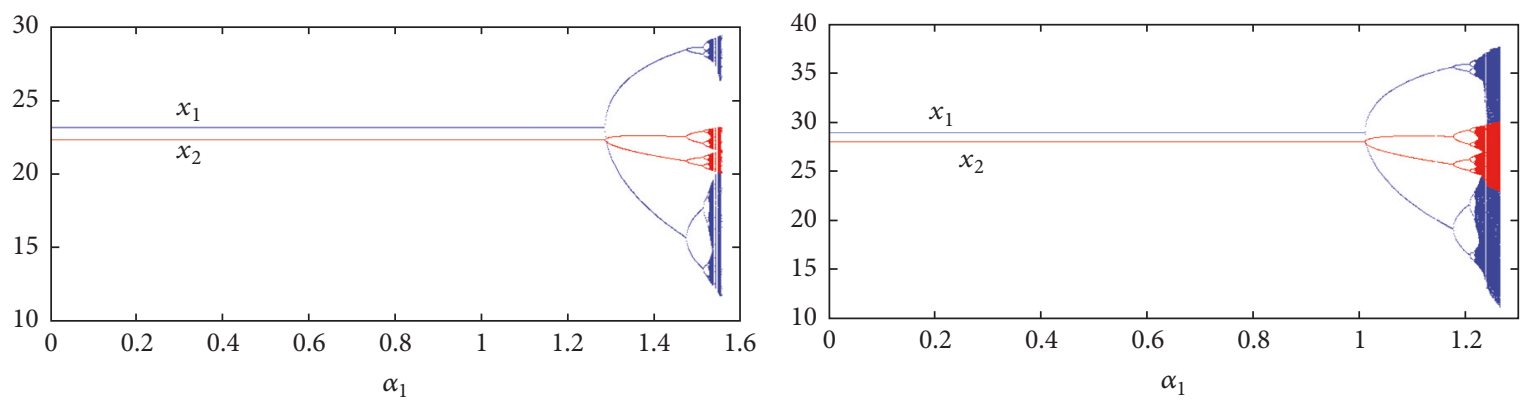

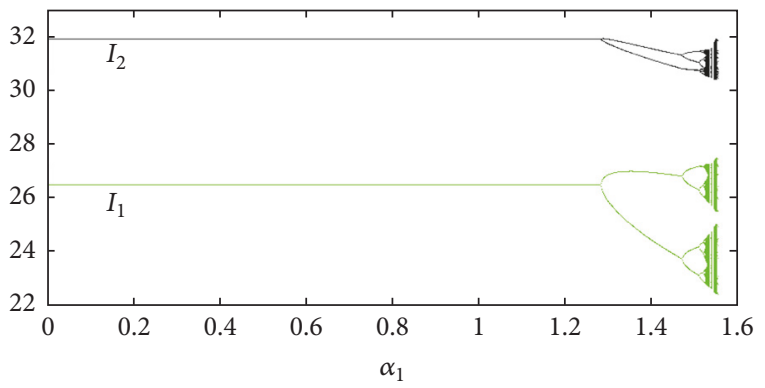

(a) $P=1.5$

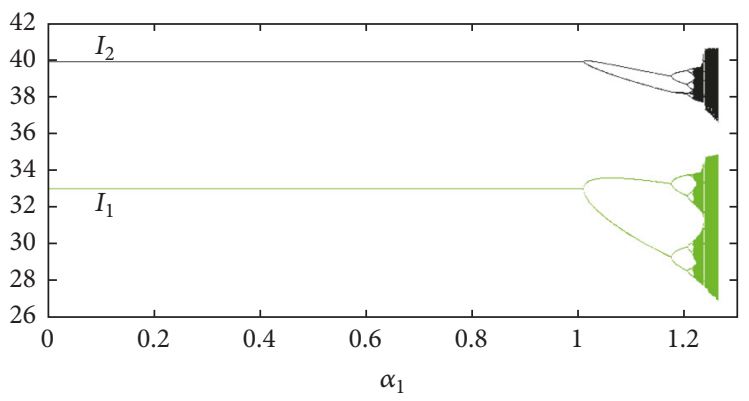

(b) $P=2$

FIGURE 1: Bifurcation diagrams for system (6) with respect to the adjustment rate $\alpha_{1}$.

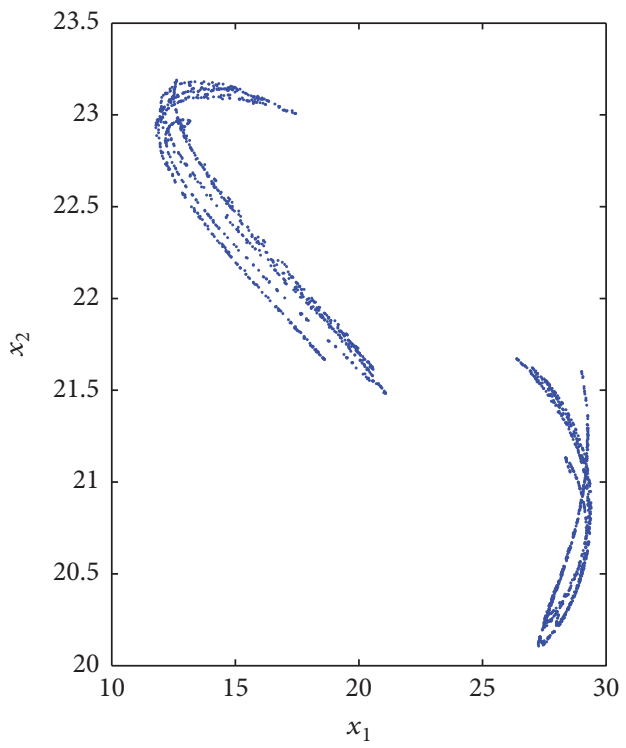

(a) $P=1.5$

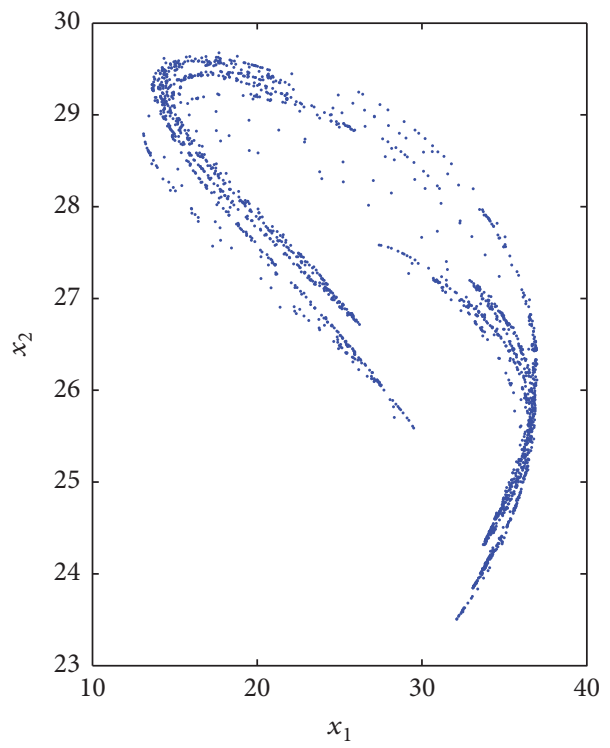

(b) $P=2$

FIGURE 2: Phase portraits for Figure 1 with various values of $P$ and $\alpha_{1}$.

(6) with the change of the model parameters, focusing on the market price of carbon trading Pand adjusting speed $\alpha$ 's influence on the dynamic nature of the system. Our study assumes that the parameters are taken as follows: $a=5, b=$ $1, \beta_{1}=0.4, \beta_{2}=0.5, k_{1}=0.4, k_{2}=0.6, B_{1}=0.6, B_{2}=$ $0.8, c_{1}=0.3, c_{2}=0.5, \theta=0.35$.

Fixed enterprise 2's strategy adjustment speed is $\alpha_{2}=0.3$. Figure 1 shows the bifurcation diagram of system (6) with enterprise 1 adjusting the speed $\alpha_{1}$, and the carbon trading market price is $P$, and $P$ is 1.5 and 2 , respectively. It is easy to see that, in Figures 1(a) and 1(b), with the value $\alpha_{1}$ increase, the system from the internal equalization changes period doubling bifurcation and eventually enters the chaotic state. Figure 1 not only shows the different paths of chaos in the system, but also shows that the larger the market price of carbon trading $P$ is, the earlier the state variable orbit enters into the chaotic state, and the system has the stronger instability. Figure 2 gives a more detailed description 


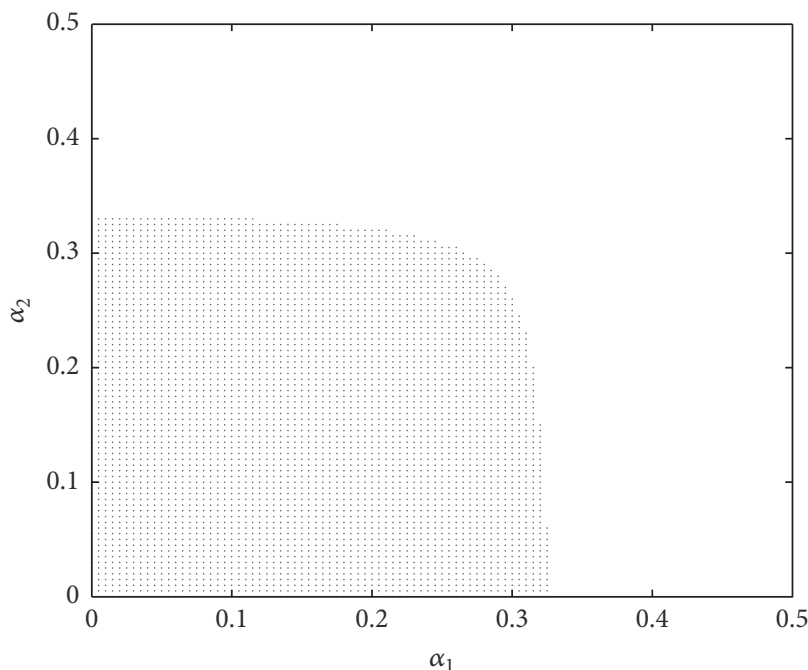

(a) $P=1.5$

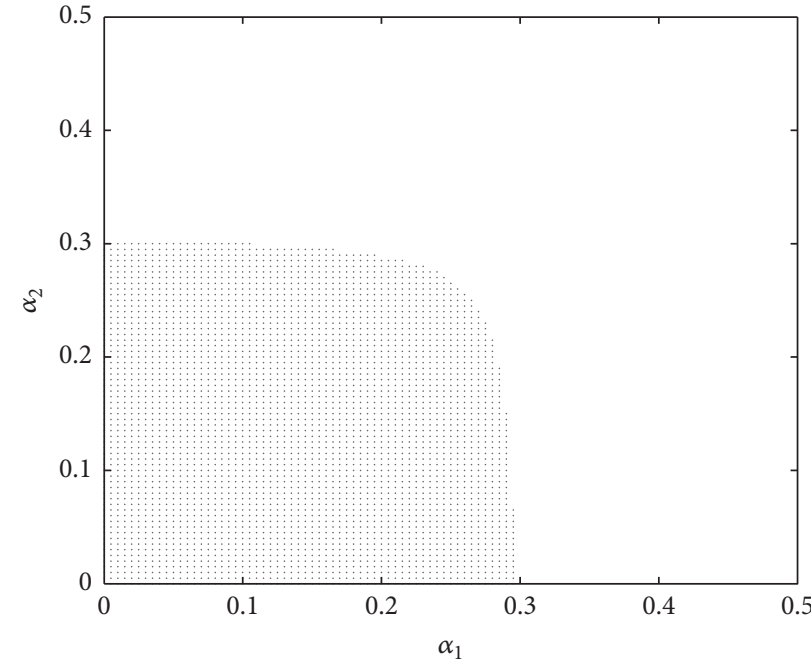

(b) $P=2$

FIGURE 3: The stability region of dynamic system $(6)$ in $\left(\alpha_{1}, \alpha_{2}\right)$-plane.
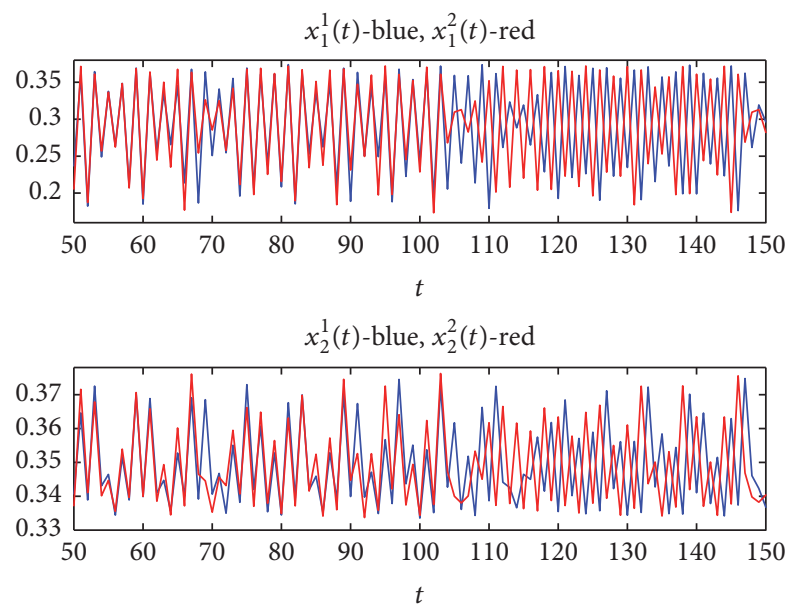

(a)
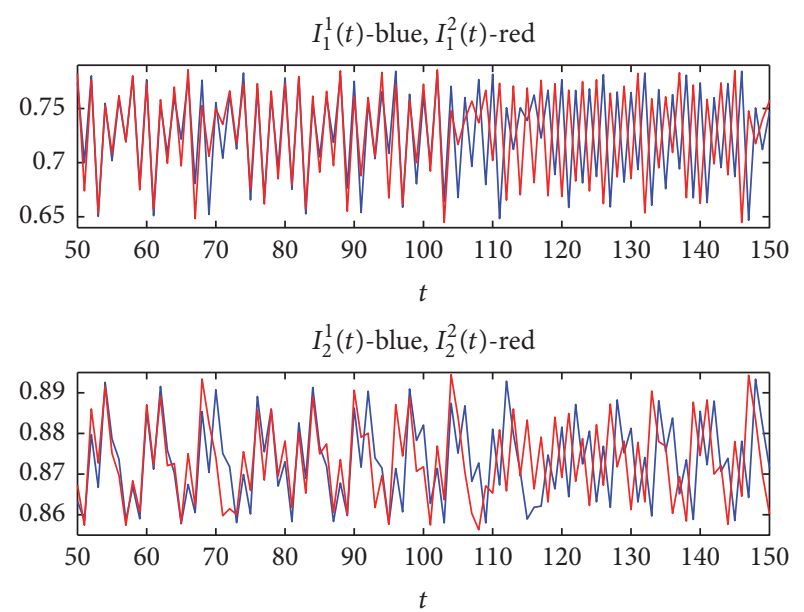

(b)

FIGURE 4: Sensitivity of the dynamic system (6) to initial conditions when losing stability.

of the system orbit's change, which is a two-dimensional phase diagram corresponding to the values of the orbit in the different states in Figure 1.

Figure 3 clearly validates this conclusion by calculating the equilibrium point stability condition (17) through the program and plotting the stable region of the system on the plane $\left(\alpha_{1}, \alpha_{2}\right)$. Comparing the two subgraphs of Figure 3, it can be seen that the increase of $P$ leads to the decrease in the stability of system (6), and the dynamic evolution of the system becomes more unstable.

We selected the adjustment coefficient $\alpha_{1}=1.23$ of the system in the chaotic state of Figure 1(b). The initial values of the system are $\left(x_{1}(0), x_{2}(0), I_{1}(0), I_{2}(0)\right)=(0.2,0.2,0.2,0.2)$ and $(0.20001,0.2,0.2,0.2)$, and the evolution diagram of the system state variables with the time is plotted. The partial image is shown in Figure 4. As the number of iterations increases, the same state variable is gradually separated under the influence of two initial conditions and subsequently conducts motion according to the respective orbit; thus, system (6) has sensitive dependence on the initial condition.

In order to highlight the effect of the market price of carbon trading on the stability of the orbital motion of the dynamical system (6), the bifurcation results of the system with different adjustment coefficients $\alpha_{1}$ are depicted in Figure 5. Figure 5 shows the doubling cycle bifurcation phenomenon. From Figure 5, the system becomes more 

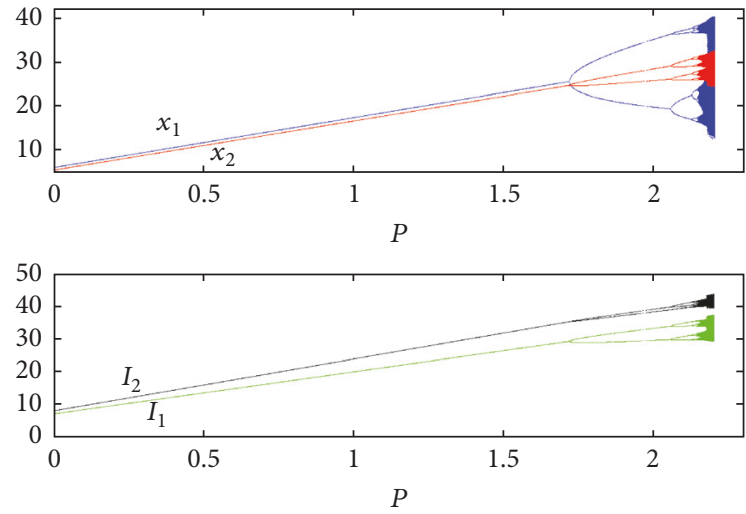

(a) $\alpha_{1}=1.15$
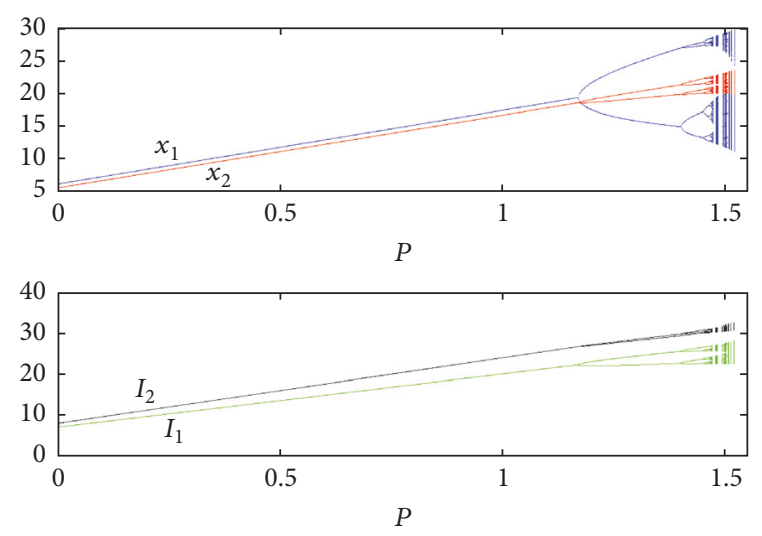

(b) $\alpha_{1}=1.55$

FIGURE 5: Bifurcation diagram for dynamic system (6) with respect to the residual rate $P$.

unstable with the increase of $P$. This suggests that increases in carbon trading's market prices lead to the increase of carbon stocks on the market, hence, facilitating environmental protectionism mechanisms which are environmentally beneficial. Companies are also actively working for energysaving and emission reduction. When the price continues to rise, it results in the increase of inventories in the market. However, beyond a certain extent, the carbon stocks create market's oversupply, hence influencing the stability of the market. Although, from an economic point of view, this phenomenon is in line with the laws of the market, this phenomenon depicts clearly an undesirable infinite loop under the harmonious development and environmental protection as it fails to achieve the desired environmental protection.

\section{Chaos Control}

From the numerical simulation results of the evolution process in system (6), it can be seen that the adjustment speed and the marketing price of carbon trading have a great influence on the stability of the system. The chaotic behavior of the dynamical system (6) is controlled by the delayed feedback control method, so that the system can be restored to the stable motion state.
Adding the feedback control item $K\left(x_{1}(t)-x_{1}(t+1)\right)$ to system (6) and simplifying the system, we obtained the following form of controlled dynamical system:

$$
\begin{aligned}
& x_{1}(t+1)=x_{1}(t)+\frac{\alpha_{1}}{1+K} x_{1}(t)[P \\
& \left.+\beta_{1}\left(\begin{array}{c}
-b B_{1} B_{2}\left(\theta I_{2}(t)+\beta_{2} x_{2}(t)\right)+a B_{1}-c_{1} B_{1} \\
-P k_{1} B_{1}-1-2 b B_{1}^{2}\left(\theta I_{1}(t)+\beta_{1} x_{1}(t)\right)
\end{array}\right)\right] \\
& x_{2}(t+1)=x_{2}(t)+\alpha_{2} x_{2}(t)[P \\
& \left.+\beta_{2}\left(\begin{array}{l}
-b B_{1} B_{2}\left(\theta I_{1}(t)+\beta_{1} x_{1}(t)\right)+a B_{2}-c_{2} B_{2} \\
-P k_{2} B_{2}-1-2 b B_{2}^{2}\left(\theta I_{2}(t)+\beta_{2} x_{2}(t)\right)
\end{array}\right)\right] \\
& I_{1}(t+1)=\theta I_{1}(t)+\beta_{1} x_{1}(t) \\
& I_{2}(t+1)=\theta I_{2}(t)+\beta_{2} x_{2}(t) .
\end{aligned}
$$

The stability of the controlled system (18) is analyzed. It is easy to know that the controlled system has the same equilibrium point as the discrete dynamical system (6). The Jacobian matrix corresponding to the controlled system (18) is

$$
J\left(x_{1}, x_{2}, I_{1}, I_{2}\right)=\left(\begin{array}{cccc}
1-\frac{\alpha_{1} M_{1}}{1+K} & \frac{-b B_{1} B_{2} x_{1} \alpha_{1} \beta_{1} \beta_{2}}{1+K} & \frac{-2 b \theta B_{1}^{2} x_{1} \alpha_{1} \beta_{1}}{1+K} & \frac{-b \theta B_{1} B_{2} x_{1} \alpha_{1} \beta_{1}}{K+1} \\
-b B_{1} B_{2} x_{2} \alpha_{2} \beta_{1} \beta_{2} & 1-\alpha_{2} M_{2} & -b \theta B_{1} B_{2} x_{2} \alpha_{2} \beta_{2} & -2 b \theta B_{2}^{2} x_{2} \alpha_{2} \beta_{2} \\
\beta_{1} & 0 & \theta & 0 \\
0 & \beta_{2} & 0 & \theta
\end{array}\right) .
$$

As shown in Figure 1(b), when the adjustment coefficient is about 1.21 , system (6) enters the chaotic state. The expression of the Jacobian matrix (19) in the interior point equalization $E^{*}$ is given under the original parameter value: 

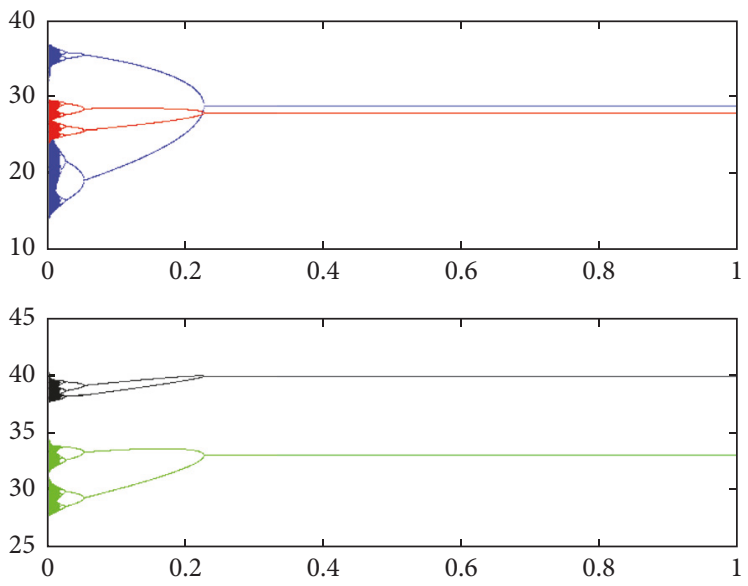

Figure 6: Bifurcation diagram of system (18) with respect to the controlling factor $k$.

$$
=\left(\begin{array}{cccc}
1-\frac{1.37453}{1+K} & -\frac{0.68728}{1+K} & -\frac{1.20272}{1+K} & -\frac{0.48109}{1+K} \\
-0.1261 & 0.7478 & -0.11034 & -0.17654 \\
0.4 & 0 & 0.35 & 0 \\
0 & 0.5 & 0 & 0.35
\end{array}\right) .
$$

Our study sets the characteristic polynomial of the matrix (20) as $P(\lambda)=\lambda^{4}+u_{1} \lambda^{3}+u_{2} \lambda^{2}+u_{3} \lambda+u_{4}$, and when the coefficients of the matrix satisfy the Schur-Cohn criterion, then

$$
\begin{aligned}
& \left|1+u_{2}+u_{4}\right|>u_{1}+u_{3} \\
& 1 \pm u_{4}>0 \\
& \left|1-u_{4}^{2}+u_{2} u_{4}-u_{2} u_{4}^{2}+u_{1} u_{3} u_{4}-u_{3}^{2}\right| \\
& \quad>u_{2}-u_{2} u_{4}+u_{4}-u_{4}^{3}-u_{1} u_{3}+u_{1}^{2} u_{4} .
\end{aligned}
$$

The characteristic root of matrix (18) is located within the unit circle on the complex plane, and it can be seen that the internal equilibrium point of the controlled system (18) is stable under the settled parameter values, and the chaotic motion of system (6) can also be adjusted to the expected stable orbit, and it can consequently reach the value range of feedback gain intensity.

The effectiveness of control on the chaos of the discrete dynamical system (6) is reached by numerical simulation. Figure 6 shows that if we add the feedback control term $K\left(x_{1}(t)-x_{1}(t+1)\right)$ into the dynamic iterative equation on the emission reduction of the controlled system (6), the unstable orbit can be controlled as long as the feedback gain intensity $K$ is greater than 0.231 . The process that chaotic behaviors of the controlled system under the different gain intensities evolve from the initial value to the stable orbit is expressed in Figure 7. By comparison, the time the state variable reaches stable state in Figure 7(b) is shorter than that in Figure 7 (a). The carbon trading market also gets stabilized more quickly.

\section{Conclusion}

Based on the hypothesis of participant's bounded rationality, our study formulated a novel Cournot duopoly game model of carbon emission reduction and subsequently analyzed the dynamic adjustment mechanism of emission reduction of the participating enterprises. The main idea in our model is that each firm's decision is to choose its carbon emission in each period according to the marginal profit observed from the previous period. We have established a corresponding dynamics of players' carbon emission adjustment and done a detailed dynamic analysis for it. There are three boundary equilibriums and a unique interior equilibrium in this system. By analyzing the stability of each equilibrium point of the discrete dynamical model system, the conditions of the asymptotic equilibrium of the interior point were obtained according to the Schur-Cohn stability criterion. Furthermore, the numerical simulation model of this paper intuitively shows the dynamic evolution process and the complexity of the system. The simulation results revealed that the parameters influence the dynamic properties of the system. It is observed that the equilibrium of the system may lose stability via different bifurcations, either flip bifurcation or NeimarkSacker bifurcation. Moreover, when the adjustment speed gets too high, the system is likely to lose the original stability and even show complex chaos easily. Higher carbon trading market prices affect the stability of the system and may lead to system instability. Finally, the delay control method has been proved to effectively control the system in the quest to restore back a stable and an equilibrium market.

\section{Conflicts of Interest}

The author declares that they have no conflicts of interest. 


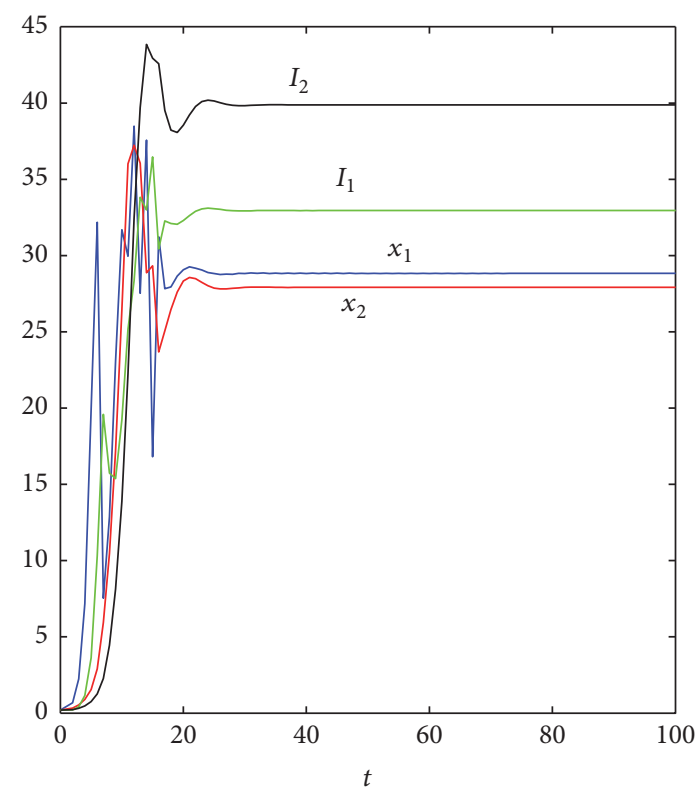

(a) $K=0.21$

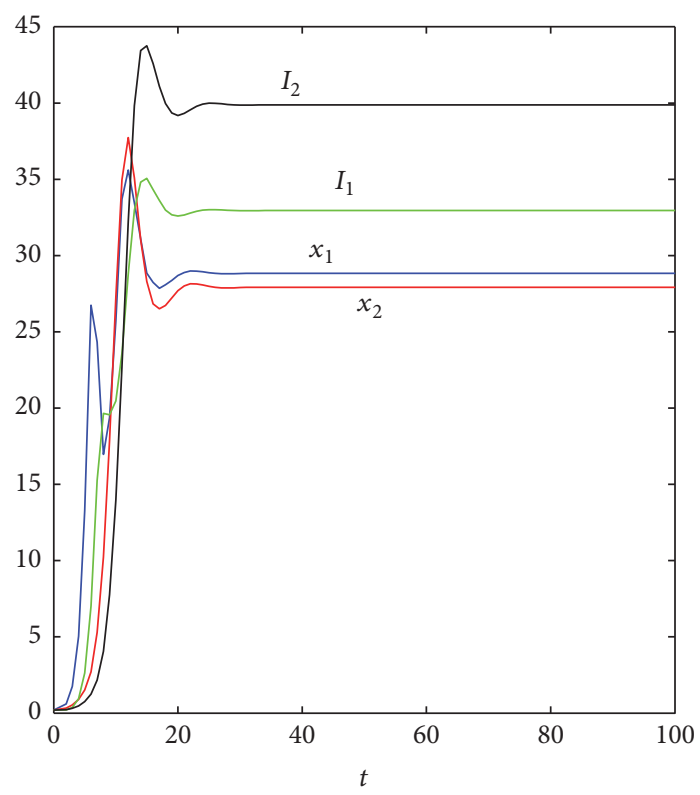

(b) $K=0.45$

FIgURE 7: The evolution path of system (18) at different gain intensities.

\section{Acknowledgments}

This work is supported by the National Nature Science Foundation of China (nos. 71171099, 71471076, and 71701090), China Scholarship Council under Grant 20123227110011, and Postgraduate Research \& Practice Innovation Program of Jiangsu Province (KYCX17_1743).

\section{References}

[1] C. Wenying and W. U. Zongxin, "Carbon emission permit al location and trading," Journal of Tsinghua University (Science and Technology), vol. 38, no. 12, pp. 15-18, 1998.

[2] L. H. Goulder, M. A. C. Hafstead, and M. Dworsky, "Impacts of alternative emissions allowance allocation methods under a federal cap-and-trade program," Journal of Environmental Economics and Management, vol. 60, no. 3, pp. 161-181, 2010.

[3] C. Xiao-hong and W. Zhi-yun, "Empirical Research on Price Impact Factor of Carbon Emission Exchange," Evidence from EU ETS. Systems Engineering, vol. 30, no. 2, pp. 53-60, 2012.

[4] M. Kara, S. Syri, A. Lehtilä et al., "The impacts of EU CO2 emissions trading on electricity markets and electricity consumers in Finland," Energy Economics, vol. 30, no. 2, pp. 193-211, 2008.

[5] A. Cournot, The American Mathematical Monthly, Mathematical Association of America, Washington, DC, USA, 1928.

[6] F. Tramontana, A. A. Elsadany, B. Xin, and H. N. Agiza, "Local stability of the Cournot solution with increasing heterogeneous competitors," Nonlinear Analysis: Real World Applications, vol. 26, pp. 150-160, 2015.

[7] J. Bertrand, "Révue de la Théorie Mathématique de la Richesse Sociale et des Recherches sur les Principles Mathématiques de la Théorie des Richesses," Journal des Savants, pp. 499-508, 1883.

[8] E. Ahmed, A. A. Elsadany, and T. Puu, "On Bertrand duopoly game with differentiated goods," Applied Mathematics and Computation, vol. 251, pp. 169-179, 2015.
[9] A. A. Elsadany, H. N. Agiza, and E. M. Elabbasy, "Complex dynamics and chaos control of heterogeneous quadropoly game," Applied Mathematics and Computation, vol. 219, no. 24, pp. 11110-11118, 2013.

[10] J. n. Andaluz and G. Jarne, "On the dynamics of economic games based on product differentiation," Mathematics and Computers in Simulation, vol. 113, pp. 16-27, 2015.

[11] Z. Ding, Q. Li, S. Jiang, and X. Wang, "Dynamics in a Cournot investment game with heterogeneous players," Applied Mathematics and Computation, vol. 256, pp. 939-950, 2015.

[12] X. X. Liao, The stability of power system theory and application, National defence industry press, Beijing, China, 2000. 


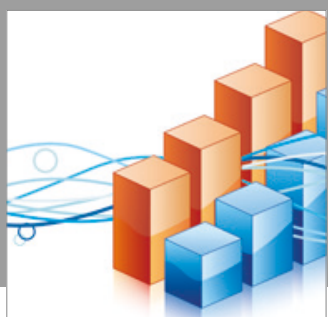

Advances in

Operations Research

vatersals

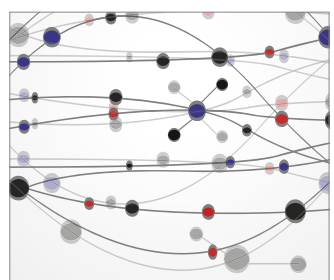

\section{The Scientific} World Journal
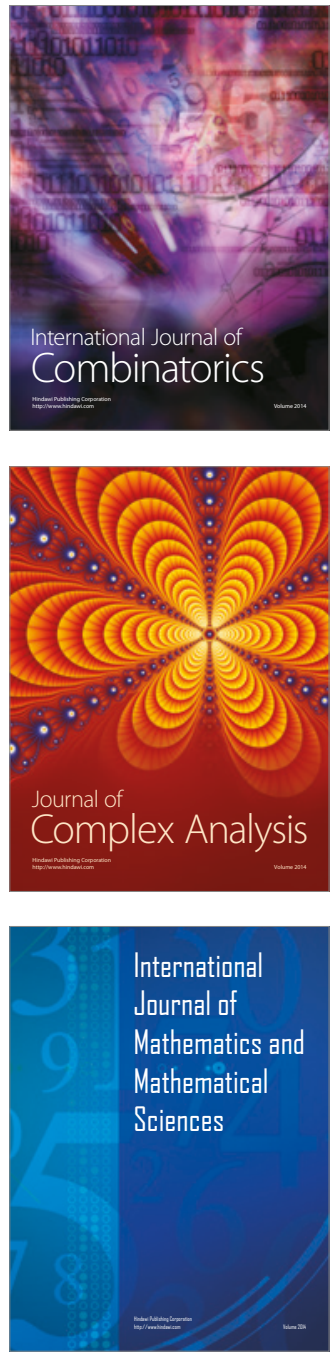
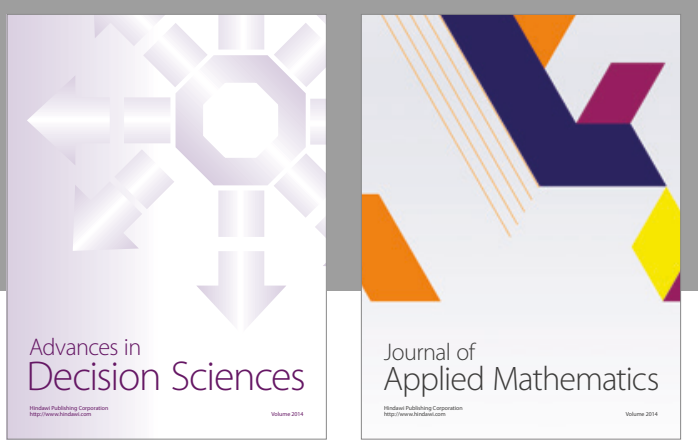

Algebra

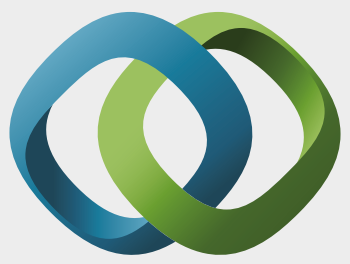

\section{Hindawi}

Submit your manuscripts at

https://www.hindawi.com
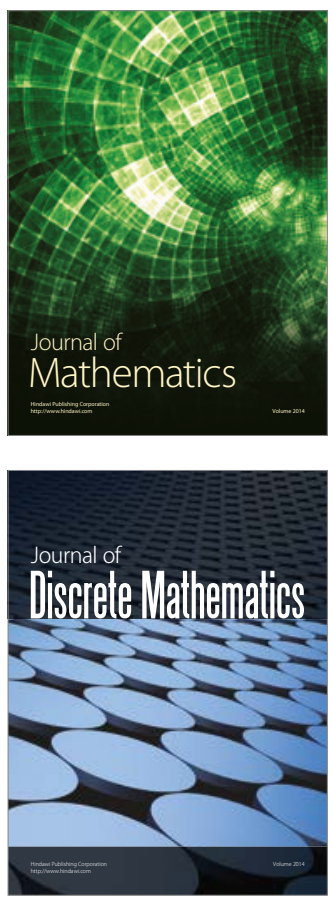

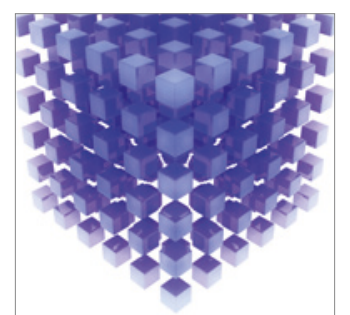

Mathematical Problems in Engineering
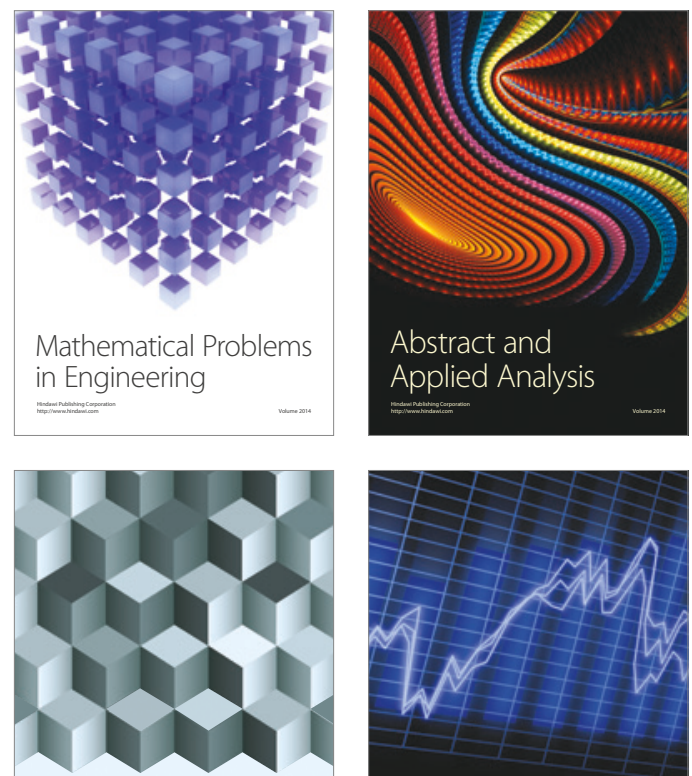

Journal of

Function Spaces

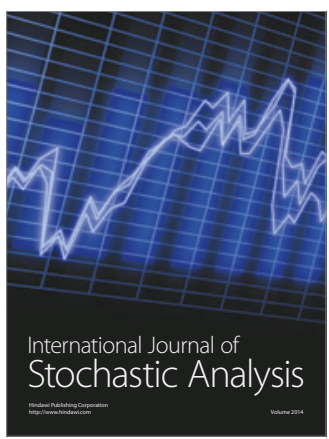

Probability and Statistics
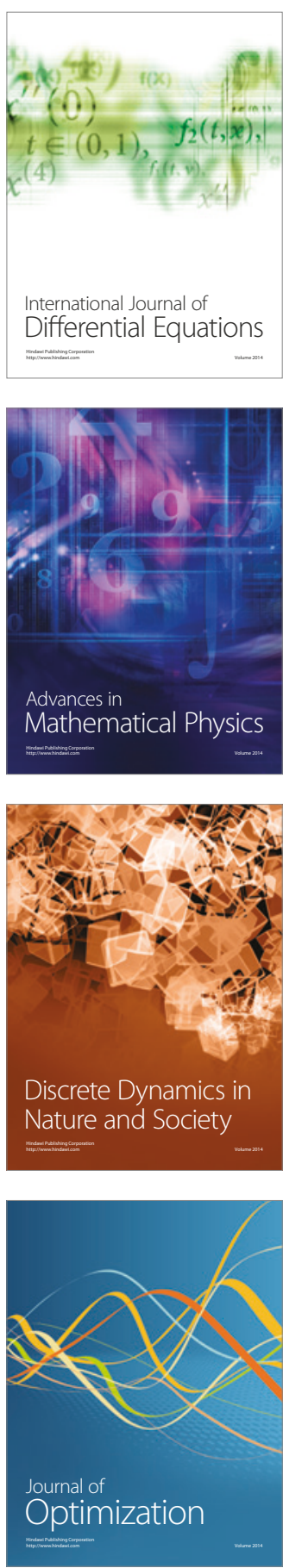